\section{Physiological Assessment of Cool-season Turfgrasses Under Ultraviolet-B Stress}

\author{
Xunzhong Zhang ${ }^{1}$ and Erik H. Ervin \\ Department of Crop and Soil Environmental Sciences, Virginia Polytechnic \\ Institute and State University, 429 Smyth Hall, Blacksburg, VA 24061-0404
}

Additional index words. chlorophyll fluorescence, electrolyte leakage, sod, superoxide dismutase

\begin{abstract}
Ultraviolet-B $(280-320 \mathrm{~nm})$ radiation is one of the major factors causing quality decline of transplanted turfgrass sod. Information on physiological parameters for assessing turfgrass tolerance to ultraviolet-B stress is lacking. The objective of this study was to evaluate ultraviolet- $B$ tolerance of four cool-season turfgrass species and varieties using selected physiological parameters under artificial ultraviolet-B radiation stress. The physiological performance of 18 varieties of tall fescue (TF; Festuca arundinacea Schreb.), chewings fescue (CF; Festuca rubra L. ssp. commutata Gaud.), perennial ryegrass (PRG; Lolium perenne L.), and kentucky bluegrass (KBG; Poa pratensis L.) were subjected to continuous, artificial ultraviolet-B radiation $\left(70 \mu \mathrm{mol} \cdot \mathrm{m}^{-2} \cdot \mathrm{s}^{-1}\right)$ for 10 days. Visual quality ratings of TF, CF, PRG, and KBG measured at Day 10 were reduced by $49 \%, 18 \%, 51 \%$, and $74 \%$, respectively, relative to that at trial initiation. Ultraviolet$B$ tolerance in CF was the greatest, KBG was the least, and TF and PRG were intermediate. 'Ebony' was the most ultraviolet-B-tolerant TF variety, whereas 'BlueTastic', 'BlueRiffic', and ' 747 ' had greater ultraviolet-B tolerance than the other four KBG varieties. No differences were observed in ultraviolet-B tolerance between varieties in either CF or PRG. The ultraviolet-B-tolerant species had less electrolyte leakage (EL), greater canopy photochemical efficiency (PEc), and relatively smaller and slower upregulation in antioxidant superoxide dismutase (SOD) activity relative to ultravioletB-sensitive ones. The results suggest that EL, PEc, and SOD may be used as physiological parameters in selecting ultraviolet-B-tolerant species and varieties for sod production and lawn establishment.
\end{abstract}

Cool-season turfgrass species such as kentucky bluegrass (KBG) and tall fescue (TF) are widely used for sod production, lawns, and athletic fields in the United States and other temperate parts of the world. Previous studies have shown that ultraviolet-B radiation stress is one of the major factors causing quality decline of newly transplanted turfgrass (Ervin et al., 2004a). Exposure of the heat-stressed turfgrass sod to high levels of ultraviolet-B $(280-320 \mathrm{~nm})$ radiation at replanting causes photo-oxidative stress and further decreases the potential for posttransplant sod survival (Ervin et al., 2004b; Heckman et al., 2001; King et al., 1982; Zhang et al., 2003).

High-intensity ultraviolet-B irradiation may damage the photosynthetic system and other metabolic processes of plants (Costa et al., 2002; Schmidt and Zhang, 2001; Teramura and Ziska, 1996; Tevini and Teramura, 1989). Ultraviolet-B irradiation has been shown to decrease the activity of the photosystem II complex with a corresponding

Received for publication 21 Apr. 2009. Accepted for publication 3 June 2009.

We thank Jonathan Green Company for the financial support of this research.

${ }^{1}$ To whom reprint requests should be addressed; e-mail xuzhang@vt.edu. decrease in electron transport and ATP synthesis, ultimately resulting in oxidative damage (Bjorn, 2004; Rao et al., 1996). High ultraviolet-B may also affect photosynthesis indirectly by photobleaching and photodegradation of pigments (Strid and Porra, 1992; Takeuchi et al., 1995; Zhang et al., 2005). As a result, cell membranes are damaged and turfgrass quality and photochemical activity are significantly reduced (Schmidt and Zhang, 2001; Zhang et al., 2005).

Plants possess various means of tolerating or avoiding ultraviolet-B stress (Caldwell et al., 1983). Antioxidants are important ultravioletB plant defense mechanisms. Antioxidants such as superoxide dismutase (SOD) function to scavenge reactive oxygen species (ROS) and protect plants from ultraviolet-B-induced oxidative damage (Bornman et al., 1997; Rao et al., 1996; Smirnoff, 1995; Teramura and Ziska, 1996; Zhang et al., 2005). Previous studies have shown that ultraviolet-B tolerance of KBG is closely associated with levels of leaf antioxidants and pigments (Ervin et al., 2004b; Zhang et al., 2005).

Plant tolerance to ultraviolet-B stress varies greatly among species and varieties within the same species. Some species are particularly sensitive and show signs of damage at moderate increases in ultraviolet-B, whereas other species show no response (Tevini and Teramura, 1989). These differences are also found between varieties of the same species (Teramura et al., 1990). In a study with KBG, Zhang et al. (2005) noted that the variety 'Moonlight', containing higher levels of antioxidants and pigments, had greater resistance to ultraviolet-B stress relative to the variety 'Limerick' with less relative antioxidant and pigment levels.

Hundreds of varieties from several coolseason turfgrass species have been released and are available for sod production, lawns, and athletic fields. Wide variation exists among these species and varieties in visual quality and tolerance to environmental stresses (National Turfgrass Evaluation Program, 2008). Because ultraviolet-B radiation has been identified as a major factor causing turfgrass quality decline, it is possible that turfgrass species and varieties could be assessed physiologically using ultraviolet-B as a stressor in controlled environments (Ervin et al., 2004b). Few physiological assessments, however, of different cool-season turfgrass species and varieties for ultraviolet-B tolerance have been reported. The objectives of this study were to evaluate performance and physiological fitness of selected varieties of $\mathrm{TF}$, chewings fescue $(\mathrm{CF})$, perennial ryegrass (PRG), and KBG under artificial ultraviolet-B stress and to provide physiological information for selecting ultraviolet-B tolerant coolseason turfgrass species and varieties.

\section{Materials and Methods}

Turfgrass culture and ultraviolet- $B$ treatment. This study was conducted in a greenhouse facility at Virginia Tech, Blacksburg, VA. A total of 18 varieties of four coolseason turfgrasses were studied: five varieties of TF; three varieties of $\mathrm{CF}$; three varieties of PRG; and seven varieties of KBG. These grasses were kindly provided by Jonathan Green Company (Greendale, NJ). Foam cups (9.5 $\mathrm{cm}$ diameter, $11 \mathrm{~cm}$ deep), with the bottom having four holes for drainage, were filled with calcined clay (Profile Products, Chicago, IL) and irrigated with one-fourth strength Hoagland's complete nutrient solution to field capacity.

Seeds were planted by hand at $9.8 \mathrm{~g} \cdot \mathrm{m}^{-2}$ (KBG), $29.3 \mathrm{~g} \cdot \mathrm{m}^{-2}(\mathrm{CF})$, and $39.1 \mathrm{~g} \cdot \mathrm{m}^{-2}$ (TF and PRG) on 27 Feb. 2007 and the cups were placed under a mist system. Nitrogen was applied to the seedlings at $2.5 \mathrm{~g} \cdot \mathrm{m}^{-2}$ with a $20 \mathrm{~N}-8.8 \mathrm{P}-16.6 \mathrm{~K}$ soluble fertilizer every 2 weeks after establishment. The grass was trimmed weekly to $5 \mathrm{~cm}$.

Fifty-three days after planting, the plants were removed from the mist system and placed in trays $(60 \times 100 \times 6 \mathrm{~cm})$ with water ( $1 \mathrm{~cm}$ deep) and subjected to ultraviolet-B radiation stress for $10 \mathrm{~d}$ (from $23 \mathrm{Apr}$. through 3 May). Briefly, the varieties were placed under artificial ultraviolet-B $\left(70 \mu \mathrm{mol} \cdot \mathrm{m}^{-2} \cdot \mathrm{s}^{-1}\right)$ provided by three $40-\mathrm{W}$ ultraviolet-B fluorescent lamps (UVB-313; Q-Panel Laboratory Products, Cleveland, $\mathrm{OH}$ ). This ultraviolet-B intensity has proved appropriate for identifying stress tolerance of different varieties/ treatments in a relatively short period of time 
(Ervin et al., 2004b). The containers were spaced evenly $(2.5 \mathrm{~cm})$, kept $0.5 \mathrm{~m}$ below the ultraviolet-B source, and grown under continuous ultraviolet-B irradiation in a greenhouse maintained at $23 / 20{ }^{\circ} \mathrm{C}$ (day/night). The photoperiod averaged $11 \mathrm{~h}$. The containers were moved around the bench space weekly. The grasses were irrigated three times a week to prevent moisture stress. Total ultraviolet flux (250 to $400 \mathrm{~nm}$ ) from the fluorescent lamps was measured with an ultraviolet meter (Apogee Instruments, Inc., Logan, UT) and was uniform on the bench space area. The meter was placed vertically with the sensor $0.5 \mathrm{~m}$ below the ultraviolet-B source. On 3 May, the plugs were removed from ultraviolet-B treatment and placed under a mist system for recovery from 3 May to 18 May. Turfgrass quality ratings and chlorophyll fluorescence readings took place on Days 1, 5, 10, and 25 after ultraviolet-B initiation. On the same days, leaf samples were collected for analysis of electrolyte leakage (EL) and SOD activity. The samples for SOD were immediately frozen with liquid nitrogen and stored at $-80^{\circ} \mathrm{C}$ until laboratory analysis could be performed.

Turfgrass quality rating. Turfgrass quality or injury was rated based on a visual scale of 1 to 9 with 9 indicating the best quality or no injury and 1 indicating the worst quality or most injury (chlorosis).

Canopy photochemical efficiency measurement. The photochemical efficiency (PEc) was measured indirectly by chlorophyll fluorescence using a dual-wavelength fluorometer (OS-50; Opti-Sciences, Inc., Tyngsboro, MA). Photochemical efficiency $(\mathrm{Fv} / \mathrm{Fm}$, that is, a ratio of variable fluorescence to maximum fluorescence at $690 \mathrm{~nm}$ ) was determined from chlorophyll fluorescence signals (Bolhar-Nordenkampf and Oquist, 1993; Zhang et al., 2005).

Leaf electrolyte leakage. Leaf blades (50 $\mathrm{mg}$ ) were cut into $3-\mathrm{mm}$ sections and transferred to a $50-\mathrm{mL}$ centrifuge tube containing $20 \mathrm{~mL}$ of deionized distilled water. The samples were placed in a shaker for $12 \mathrm{~h}$ at $25^{\circ} \mathrm{C}$. After an initial electrical conductivity $\left(\mathrm{EC}_{1}\right)$ reading using a conductivity meter, the samples were autoclaved at $120{ }^{\circ} \mathrm{C}$ for 30 min. After cooling, a second EC $\left(\mathrm{EC}_{2}\right)$ reading was taken. The EL (\%) is expressed as $\left(\mathrm{EC}_{1} / \mathrm{EC}_{2}\right) \times 100$.

Leaf superoxide dismutase activity. Frozen leaf samples $(100 \mathrm{mg}$ ) were crushed with liquid nitrogen and extracted with a pestle in an ice-cold mortar with $3 \mathrm{~mL}$ of $0.05 \mathrm{M}$ $\mathrm{Na}_{2} \mathrm{HPO}_{4} / \mathrm{NaH}_{2} \mathrm{PO}_{4}$ (pH 7.0) buffer containing $0.2 \mathrm{~mm}$ ethylenediamine-tetra-acetic acid, and $1 \%$ polyvinyl-pyrrolidone. The homogenates were centrifuged at $4{ }^{\circ} \mathrm{C}$ for $20 \mathrm{~min}$ at $13,000 \mathrm{~g}_{\mathrm{n}}$. The supernatants were collected and used for assays of enzymatic activity.

Superoxide dismutase activity was determined according to the method of Banowetz et al. (2004). The SOD activity was calculated based on changes in absorbance at 560 $\mathrm{nm}$ and from the standard curve.

Determination of protein concentration. Because SOD activity is presented on a
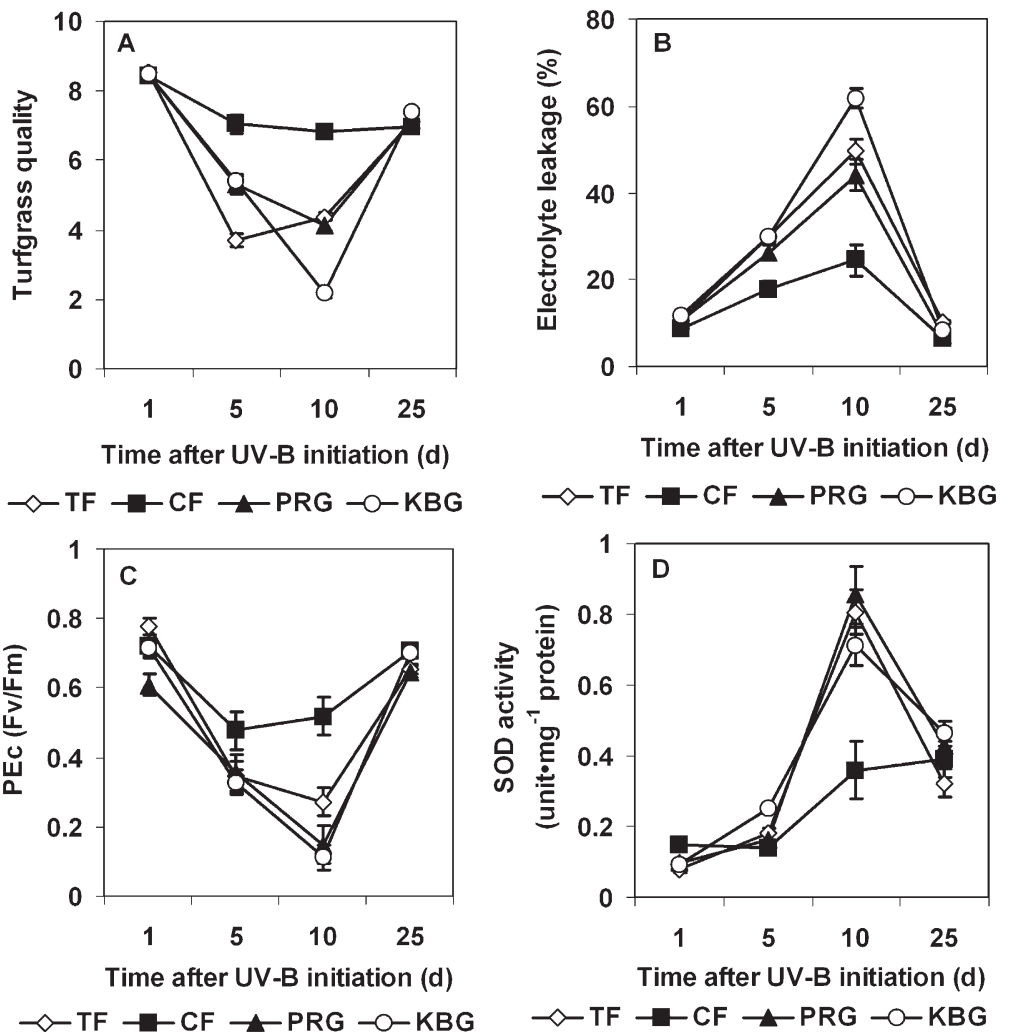

Fig. 1. Responses of turfgrass visual quality (A), leaf electrolyte leakage (EL; B), canopy photochemical efficiency ( $\mathrm{PEc} ; \mathrm{Fv} / \mathrm{Fm} ; \mathbf{C}$ ), and leaf superoxide dismutase (SOD) activity (D) to artificial ultraviolet-B radiation $\left(70 \mu \mathrm{mol} \cdot \mathrm{m}^{-2} \cdot \mathrm{s}^{-1}\right)$ stress in tall fescue $(\mathrm{TF})$, chewings fescue $(\mathrm{CF})$, perennial ryegrass $(\mathrm{PRG})$, and kentucky bluegrass (KBG). Turfgrass quality was rated on a visual scale of 1 to 9 with 9 indicating the best quality; vertical bars represent SEs at a given day; the artificial ultraviolet-B was removed at Day 10.

Table 1. Turfgrass quality, leaf electrolyte leakage (EL), canopy photochemical efficiency (PEc), and superoxide dismutase (SOD) activity of tall fescue (TF) varieties under ultraviolet-B stress.

\begin{tabular}{|c|c|c|c|c|}
\hline \multirow[b]{2}{*}{ Variety } & \multicolumn{4}{|c|}{ Time after ultraviolet-B initiation (d) } \\
\hline & 1 & 5 & $10^{\mathrm{z}}$ & 25 \\
\hline & \multicolumn{4}{|c|}{ Turfgrass quality ( $1-9$ scale; $9=$ the best) } \\
\hline Montana & $8.6 \mathrm{a}$ & $4.0 \mathrm{ab}^{\mathrm{y}}$ & $4.4 \mathrm{~b}$ & $7.4 \mathrm{a}$ \\
\hline Dakota & $8.5 \mathrm{a}$ & $2.9 \mathrm{c}$ & $4.1 \mathrm{~b}$ & $6.8 \mathrm{a}$ \\
\hline Taos & $8.5 \mathrm{a}$ & $3.5 \mathrm{~b}$ & $4.4 \mathrm{~b}$ & $7.1 \mathrm{a}$ \\
\hline Tombstone & $8.6 \mathrm{a}$ & $3.9 \mathrm{ab}$ & $4.1 \mathrm{~b}$ & $6.9 \mathrm{a}$ \\
\hline \multirow[t]{2}{*}{ Ebony } & $8.5 \mathrm{a}$ & $4.1 \mathrm{a}$ & $4.9 \mathrm{a}$ & $7.4 \mathrm{a}$ \\
\hline & \multicolumn{4}{|c|}{ Leaf EL (\%) } \\
\hline Montana & $8.7 \mathrm{~b}$ & $29.6 \mathrm{a}$ & $53.8 \mathrm{a}$ & $8.7 \mathrm{c}$ \\
\hline Dakota & $10.5 \mathrm{ab}$ & $32.2 \mathrm{a}$ & $51.6 \mathrm{a}$ & $11.1 \mathrm{a}$ \\
\hline Taos & $11.5 \mathrm{a}$ & $23.8 \mathrm{a}$ & $51.3 \mathrm{a}$ & $10.7 \mathrm{ab}$ \\
\hline Tombstone & $11.1 \mathrm{ab}$ & $32.4 \mathrm{a}$ & $52.7 \mathrm{a}$ & $11.4 \mathrm{a}$ \\
\hline \multirow[t]{2}{*}{ Ebony } & $10.7 \mathrm{ab}$ & $30.9 \mathrm{a}$ & $38.0 \mathrm{~b}$ & $9.3 \mathrm{bc}$ \\
\hline & \multicolumn{4}{|c|}{$P E c(F v / F m)$} \\
\hline Montana & $0.849 \mathrm{a}$ & $0.380 \mathrm{a}$ & $0.296 \mathrm{ab}$ & $0.613 \mathrm{~b}$ \\
\hline Dakota & $0.755 \mathrm{a}$ & $0.279 \mathrm{a}$ & $0.216 \mathrm{ab}$ & $0.647 \mathrm{ab}$ \\
\hline Taos & $0.772 \mathrm{a}$ & $0.329 \mathrm{a}$ & $0.256 \mathrm{ab}$ & $0.648 \mathrm{ab}$ \\
\hline Tombstone & $0.815 \mathrm{a}$ & $0.262 \mathrm{a}$ & $0.093 \mathrm{~b}$ & $0.684 \mathrm{a}$ \\
\hline \multirow[t]{2}{*}{ Ebony } & $0.693 \mathrm{a}$ & $0.479 \mathrm{a}$ & $0.499 \mathrm{a}$ & $0.686 \mathrm{a}$ \\
\hline & \multicolumn{4}{|c|}{ SOD activity (unit/mo protein) } \\
\hline Montana & $0.074 \mathrm{~b}$ & $0.068 \mathrm{~d}$ & $0.890 \mathrm{~b}$ & $0.460 \mathrm{a}$ \\
\hline Dakota & $0.098 \mathrm{a}$ & $0.220 \mathrm{~b}$ & $0.557 \mathrm{c}$ & $0.256 \mathrm{c}$ \\
\hline Taos & $0.063 \mathrm{~b}$ & $0.263 \mathrm{a}$ & $0.491 \mathrm{c}$ & $0.313 \mathrm{~b}$ \\
\hline Tombstone & $0.077 \mathrm{~b}$ & $0.238 \mathrm{ab}$ & $1.041 \mathrm{a}$ & $0.278 \mathrm{bc}$ \\
\hline Ebony & $0.095 \mathrm{a}$ & $0.111 \mathrm{c}$ & $1.053 \mathrm{a}$ & $0.306 \mathrm{bc}$ \\
\hline
\end{tabular}

${ }^{\mathrm{z}}$ The time of ultraviolet-B removal.

${ }^{y}$ Values followed by same letters within same column for each data set are not significantly different at $P \leq$ 0.05 . 
protein basis, protein concentration of the extract from each sample was analyzed by the bicinchoninic method with bovine serum albumin serving as the standard (Smith, 1985).

Experimental design and data analysis. A randomized complete block design was used with four replications. The data were analyzed using analysis of variance and mean separations were performed using a Fisher's protected least significance difference test $(P$ $\leq 0.05)$. The differences between species and between varieties within each species were analyzed separately. Correlations of turfgrass quality with PEc, EL, and SOD activity, and EL with PEc were analyzed (SAS Version 8e for Windows; SAS Institute Inc., 2001). Regressions between these variables were also analyzed using Sigmaplot (Version 10; Systat Software, Inc., 2007).

\section{Results}

Differences in ultraviolet-B tolerance between species. Ultraviolet-B radiation caused damage to all four species (Fig. 1A). Visual quality ratings of $\mathrm{TF}, \mathrm{CF}, \mathrm{PRG}$, and KBG measured at Day 10 were reduced by $49 \%, 18 \%, 51 \%$, and $74 \%$, respectively, relative to that at trial initiation (Fig. 1A). Ultraviolet-B radiation also reduced $\mathrm{PEc}$ and increased EL and SOD activity (Fig. 1B-D).

A significant difference in ultraviolet-B resistance was found between species (Fig. 1). Visual quality ratings and PEc were the highest in CF and the lowest in KBG at d 10. Leaf EL was the highest in KBG and lowest in CF (Fig. 1B). The activity of SOD increased in response to ultraviolet- $\mathrm{B}$ in all species (Fig. 1D).

Differences in ultraviolet- $B$ tolerance between varieties. Among TF varieties, 'Ebony' had greater ultraviolet-B tolerance than the other four varieties based on visual quality, EL, PEc, and SOD activity (Table 1).

The three $\mathrm{CF}$ varieties were similar in ultraviolet-B tolerance. A greater increase in SOD activity was measured in 'FC3' relative to 'Salisbury' and 'Hood' during ultraviolet-B stress (Table 2). The three PRG varieties were similar in ultraviolet-B tolerance (Table 3). 'PR39' may be more sensitive to ultraviolet-B stress relative to 'Frontier' and 'BMF06' because of a measured rapid increase in SOD activity and decline in PEc under ultraviolet-B stress.

Overall, KBG had less ultraviolet-B tolerance relative to the other three species (Fig. 1A). A significant difference was found between KBG varieties in ultraviolet-B tolerance (Table 4). 'BlueTastic', 'BlueRiffic', and ' 747 ' had less EL and greater PEc when compared with the other four KBG varieties. Based on quality at Day 10, the seven varieties were ranked as follows: 'BlueTastic' = 'BlueRiffic' > '747' > 'Midnight' > '748' = 'Deepblue' > 'Washington'.

Relationship of turfgrass quality with physiological parameters. A significant positive correlation was found between turfgrass quality and PEc $(r=0.9039 ; P<0.01)$, whereas significant negative correlations

Table 2. Turfgrass quality, leaf electrolyte leakage (EL), canopy photochemical efficiency (PEc), and superoxide dismutase (SOD) activity of chewings fescue (CF) varieties under ultraviolet-B stress.

\begin{tabular}{|c|c|c|c|c|}
\hline \multirow[b]{2}{*}{ Variety } & \multicolumn{4}{|c|}{ Time after ultraviolet-B initiation (d) } \\
\hline & 1 & 5 & $10^{z}$ & 25 \\
\hline & \multicolumn{4}{|c|}{ Turfgrass quality (1-9 scale; $9=$ the best) } \\
\hline $\mathrm{FC}^{\prime}{ }^{\prime}$ & $8.4 \mathrm{a}$ & $7.3 \mathrm{a}$ & $6.8 \mathrm{a}$ & $7.0 \mathrm{a}$ \\
\hline Salisbury & $8.5 \mathrm{a}$ & $6.9 \mathrm{a}$ & $6.8 \mathrm{a}$ & $6.9 \mathrm{a}$ \\
\hline \multirow[t]{2}{*}{ Hood } & $8.4 \mathrm{a}$ & $7.0 \mathrm{a}$ & $7.0 \mathrm{a}$ & $7.1 \mathrm{a}$ \\
\hline & \multicolumn{4}{|c|}{ Leaf EL $(\%)$} \\
\hline $\mathrm{FC}^{\prime}{ }^{\prime}$ & $9.2 \mathrm{a}$ & $18.3 \mathrm{a}$ & $24.4 \mathrm{a}$ & $6.4 \mathrm{a}$ \\
\hline Salisbury & $8.1 \mathrm{a}$ & $18.5 \mathrm{a}$ & $23.5 \mathrm{a}$ & $6.7 \mathrm{a}$ \\
\hline \multirow[t]{2}{*}{ Hood } & $9.0 \mathrm{a}$ & $17.2 \mathrm{a}$ & $25.5 \mathrm{a}$ & $6.4 \mathrm{a}$ \\
\hline & \multicolumn{4}{|c|}{$P E c(F v / F m)$} \\
\hline $\mathrm{FC}^{\prime}$ & $0.751 \mathrm{ab}^{\mathrm{y}}$ & $0.418 \mathrm{a}$ & $0.461 \mathrm{a}$ & $0.708 \mathrm{a}$ \\
\hline Salisbury & $0.658 \mathrm{~b}$ & $0.495 \mathrm{a}$ & $0.615 \mathrm{a}$ & $0.702 \mathrm{a}$ \\
\hline \multirow[t]{2}{*}{ Hood } & $0.755 \mathrm{a}$ & $0.521 \mathrm{a}$ & $0.480 \mathrm{a}$ & $0.702 \mathrm{a}$ \\
\hline & \multicolumn{4}{|c|}{ SOD activity (unit/mg protein) } \\
\hline $\mathrm{FC}^{\prime}$ & $0.173 \mathrm{a}$ & $0.160 \mathrm{a}$ & $0.512 \mathrm{a}$ & $0.446 \mathrm{~b}$ \\
\hline Salisbury & $0.169 \mathrm{a}$ & $0.110 \mathrm{~b}$ & $0.248 \mathrm{~b}$ & $0.167 \mathrm{c}$ \\
\hline Hood & $0.100 \mathrm{~b}$ & $0.147 \mathrm{a}$ & $0.316 \mathrm{~b}$ & $0.561 \mathrm{a}$ \\
\hline
\end{tabular}

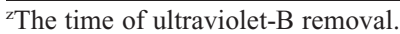

${ }^{y}$ Values followed by same letters within same column for each data set are not significantly different at $P \leq$ 0.05 .

Table 3. Turfgrass quality, leaf electrolyte leakage (EL), canopy photochemical efficiency (PEc), superoxide dismutase (SOD) activity of perennial ryegrass (PRG) varieties under ultraviolet-B stress.

\begin{tabular}{|c|c|c|c|c|}
\hline \multirow[b]{2}{*}{ Variety } & \multicolumn{4}{|c|}{ Time after ultraviolet-B initiation (d) } \\
\hline & 1 & 5 & $10^{z}$ & 25 \\
\hline & \multicolumn{4}{|c|}{ Turfgrass quality (1-9 scale; $9=$ the best) } \\
\hline Frontier & $8.5 \mathrm{a}$ & $5.6 \mathrm{a}^{\mathrm{y}}$ & $4.4 \mathrm{a}$ & $7.1 \mathrm{~b}$ \\
\hline PR39 & $8.5 \mathrm{a}$ & $5.4 \mathrm{ab}$ & $4.1 \mathrm{a}$ & $7.4 \mathrm{a}$ \\
\hline \multirow[t]{2}{*}{ BMF06 } & $8.5 \mathrm{a}$ & $4.9 \mathrm{~b}$ & $4.0 \mathrm{a}$ & $7.1 \mathrm{~b}$ \\
\hline & \multicolumn{4}{|c|}{ Leaf EL $(\%)$} \\
\hline Frontier & $9.8 \mathrm{a}$ & $26.3 \mathrm{ab}$ & $40.6 \mathrm{a}$ & $7.4 \mathrm{a}$ \\
\hline PR39 & $9.2 \mathrm{a}$ & $30.6 \mathrm{a}$ & $43.4 \mathrm{a}$ & $7.6 \mathrm{a}$ \\
\hline \multirow[t]{2}{*}{ BMF06 } & $13.2 \mathrm{a}$ & $22.2 \mathrm{~b}$ & $48.4 \mathrm{a}$ & $7.1 \mathrm{a}$ \\
\hline & \multicolumn{4}{|c|}{$P E c(F v / F m)$} \\
\hline Frontier & $0.519 \mathrm{a}$ & $0.346 \mathrm{a}$ & $0.251 \mathrm{a}$ & $0.659 \mathrm{a}$ \\
\hline PR39 & $0.635 \mathrm{a}$ & $0.327 \mathrm{a}$ & $0.000 \mathrm{a}$ & $0.625 \mathrm{a}$ \\
\hline \multirow[t]{2}{*}{ BMF06 } & $0.673 \mathrm{a}$ & $0.391 \mathrm{a}$ & $0.193 \mathrm{a}$ & $0.655 \mathrm{a}$ \\
\hline & \multicolumn{4}{|c|}{ SOD activity (uni/mg protein) } \\
\hline Frontier & $0.092 \mathrm{~b}$ & $0.113 \mathrm{c}$ & $0.565 \mathrm{~b}$ & $0.394 \mathrm{~b}$ \\
\hline PR39 & $0.065 \mathrm{~b}$ & $0.219 \mathrm{a}$ & $1.446 \mathrm{a}$ & $0.417 \mathrm{~b}$ \\
\hline BMF06 & $0.133 \mathrm{a}$ & $0.152 \mathrm{~b}$ & $0.550 \mathrm{~b}$ & $0.475 \mathrm{a}$ \\
\hline
\end{tabular}

${ }^{\mathrm{z}}$ The time of ultraviolet-B removal.

${ }^{y}$ Values followed by same letters within same column for each data set are not significantly different at $P \leq$ 0.05 .

were observed between turfgrass quality and EL $(r=-0.8894 ; P<0.01)$, turfgrass quality and SOD activity $(r=-0.5445 ; P<$ $0.01)$, PEc and EL $(r=-0.8959 ; P<0.01)$, and PEc and SOD activity $(r=-0.5364 ; P<$ 0.01 ). Significant linear regressions were also found when comparing turfgrass quality with PEc, EL, and SOD activity and between PEc and EL (Fig. 2).

\section{Discussion}

The results of this study indicate that continuous ultraviolet-B irradiation (70 $\left.\mu \mathrm{mol} \cdot \mathrm{m}^{-2} \cdot \mathrm{s}^{-1}\right)$ stress caused significant damage to shoots as reflected in quality, PEc, and leaf EL. ultraviolet stress also caused an increase in SOD activity in a relatively short period of time $(5 \mathrm{~d})$. These results are supported by previous studies (Ervin et al., 2004b; Lingakumar et al., 1999; Schmidt and Zhang, 2001; Strid and Porra, 1992; Zhang et al., 2005). Under ultraviolet-B stress, photosynthetic electron transport is inhibited and excess energy received by chlorophyll may be released as chlorophyll fluorescence or directed to $\mathrm{O}_{2}$, generating ROS in chloroplasts. Accumulation of ROS damages proteins, DNA, and membranes through lipid peroxidation, resulting in PEc decline (Smirnoff, 1995). Photosystem II is considered as one of the most susceptible sites to ultraviolet-B damage mainly because it is rich in polyunsaturated fatty acids (Lingakumar et al., 1999; Noorudeen and Kulandaivelu, 1982). Ervin et al. (2004b) reported that ultraviolet-B stress caused a decline in turf quality, PEc, and pigments of KBG. Zhang et al. (2005) noted that SOD activity increased in response to ultraviolet-B stress.

The results also indicate significant variation exists in ultraviolet-B tolerance between species and varieties within species of some cool-season turfgrasses. Ultraviolet-B 
Table 4. Turfgrass quality, leaf electrolyte leakage (EL), canopy photochemical efficiency (PEc), and superoxide dismutase (SOD) activity of kentucky bluegrass (KBG) varieties under ultraviolet-B stress.

\begin{tabular}{|c|c|c|c|c|}
\hline \multirow[b]{2}{*}{ Variety } & \multicolumn{4}{|c|}{ Time after ultraviolet-B initiation (d) } \\
\hline & 1 & 5 & $10^{z}$ & 25 \\
\hline & \multicolumn{4}{|c|}{ Turfgrass quality (1-9 scale; $9=$ the best $)$} \\
\hline BlueTastic & $8.5 \mathrm{a}$ & $6.3 \mathrm{a}^{\mathrm{y}}$ & $3.0 \mathrm{a}$ & $7.3 \mathrm{bc}$ \\
\hline BlueRiffic & $8.5 \mathrm{a}$ & $6.5 \mathrm{a}$ & $2.9 \mathrm{a}$ & $7.7 \mathrm{a}$ \\
\hline 747 & $8.4 \mathrm{a}$ & $6.1 \mathrm{a}$ & $2.6 \mathrm{ab}$ & $7.5 \mathrm{ab}$ \\
\hline 748 & $8.5 \mathrm{a}$ & $5.5 \mathrm{~b}$ & $1.8 \mathrm{~cd}$ & $7.5 \mathrm{ab}$ \\
\hline Deepblue & $8.5 \mathrm{a}$ & $5.1 \mathrm{~b}$ & $1.8 \mathrm{~cd}$ & $7.2 \mathrm{c}$ \\
\hline Washington & $8.5 \mathrm{a}$ & $2.9 \mathrm{c}$ & $1.1 \mathrm{~d}$ & $7.3 \mathrm{bc}$ \\
\hline \multirow[t]{2}{*}{ Midnight } & $8.4 \mathrm{a}$ & $5.5 \mathrm{~b}$ & $2.0 \mathrm{bc}$ & $7.3 \mathrm{bc}$ \\
\hline & \multicolumn{4}{|c|}{ Leaf EL (\%) } \\
\hline BlueTastic & $14.8 \mathrm{a}$ & $23.3 \mathrm{~d}$ & $46.3 \mathrm{~d}$ & $8.9 \mathrm{~b}$ \\
\hline BlueRiffic & $11.7 \mathrm{ab}$ & $23.9 \mathrm{~d}$ & $47.6 \mathrm{~d}$ & $8.3 \mathrm{c}$ \\
\hline 747 & $9.7 \mathrm{~b}$ & $31.8 \mathrm{bc}$ & $39.7 \mathrm{e}$ & $9.1 \mathrm{ab}$ \\
\hline 748 & $12.0 \mathrm{ab}$ & $31.2 \mathrm{bc}$ & $65.8 \mathrm{c}$ & $6.2 \mathrm{e}$ \\
\hline Deepblue & $11.0 \mathrm{ab}$ & $34.7 \mathrm{ab}$ & $72.6 \mathrm{~b}$ & $9.5 \mathrm{a}$ \\
\hline Washington & $11.5 \mathrm{ab}$ & $38.4 \mathrm{a}$ & $93.5 \mathrm{a}$ & $7.3 \mathrm{~d}$ \\
\hline \multirow[t]{2}{*}{ Midnight } & $11.6 \mathrm{ab}$ & $27.2 \mathrm{~cd}$ & $66.5 \mathrm{c}$ & $9.0 \mathrm{~b}$ \\
\hline & \multicolumn{4}{|c|}{$P E c(F v / F m)$} \\
\hline BlueTastic & $0.706 \mathrm{abc}$ & $0.317 \mathrm{a}$ & $0.162 \mathrm{ab}$ & $0.740 \mathrm{a}$ \\
\hline BlueRiffic & $0.763 \mathrm{a}$ & $0.421 \mathrm{a}$ & $0.211 \mathrm{ab}$ & $0.758 \mathrm{a}$ \\
\hline 747 & $0.751 \mathrm{ab}$ & $0.306 \mathrm{a}$ & $0.272 \mathrm{a}$ & $0.769 \mathrm{a}$ \\
\hline 748 & $0.690 \mathrm{bc}$ & $0.416 \mathrm{a}$ & $0.004 \mathrm{~b}$ & $0.665 \mathrm{~b}$ \\
\hline Deepblue & $0.695 \mathrm{bc}$ & $0.245 \mathrm{a}$ & $0.000 \mathrm{~b}$ & $0.644 \mathrm{~b}$ \\
\hline Washington & $0.713 a b c$ & $0.259 \mathrm{a}$ & $0.112 \mathrm{ab}$ & $0.680 \mathrm{~b}$ \\
\hline \multirow[t]{2}{*}{ Midnight } & $0.685 \mathrm{c}$ & $0.336 \mathrm{a}$ & $0.023 \mathrm{~b}$ & $0.666 \mathrm{~b}$ \\
\hline & \multicolumn{4}{|c|}{ SOD activity (unit/mg protein) } \\
\hline BlueTastic & $0.117 \mathrm{a}$ & $0.202 \mathrm{~d}$ & $1.093 \mathrm{a}$ & $0.923 \mathrm{a}$ \\
\hline BlueRiffic & $0.086 \mathrm{~b}$ & $0.186 \mathrm{e}$ & $0.182 \mathrm{f}$ & $0.277 \mathrm{~d}$ \\
\hline 747 & $0.052 \mathrm{c}$ & $0.175 \mathrm{e}$ & $0.763 \mathrm{c}$ & $0.253 \mathrm{~d}$ \\
\hline 748 & $0.067 \mathrm{bc}$ & $0.226 \mathrm{c}$ & $0.816 \mathrm{bc}$ & $0.672 \mathrm{~b}$ \\
\hline Deepblue & $0.120 \mathrm{a}$ & $0.243 \mathrm{~b}$ & $0.684 \mathrm{~d}$ & $0.259 \mathrm{~d}$ \\
\hline Washington & $0.083 \mathrm{~b}$ & $0.353 \mathrm{a}$ & $0.598 \mathrm{e}$ & $0.293 \mathrm{~d}$ \\
\hline Midnight & $0.136 \mathrm{a}$ & $0.357 \mathrm{a}$ & $0.844 \mathrm{~b}$ & $0.565 \mathrm{c}$ \\
\hline
\end{tabular}

${ }^{\mathrm{z}}$ The time of ultraviolet-B removal.

${ }^{y}$ Values followed by same letters within same column for each data set are not significantly different at $P \leq 0.05$.
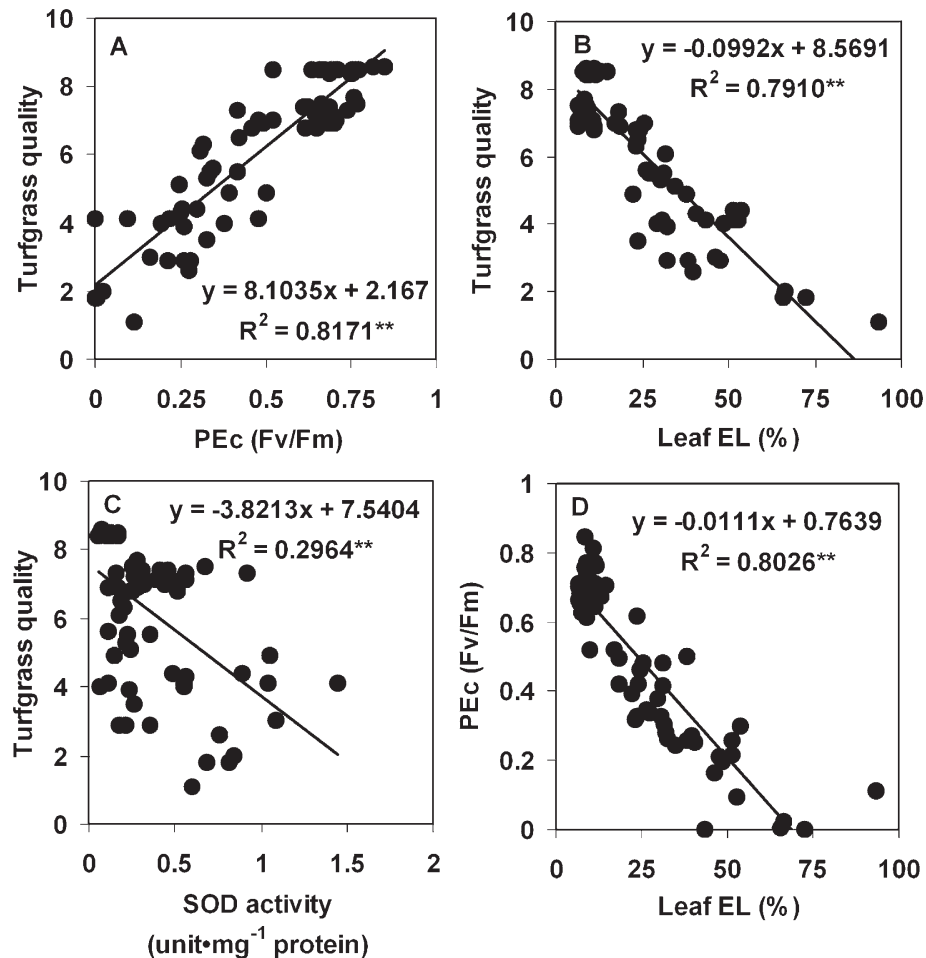

Fig. 2. Relationship of turfgrass quality with canopy photochemical efficiency (PEc; Fv/Fm; A), leaf electrolyte leakage (EL; B), leaf superoxide dismutase (SOD) activity (C), and relationship of canopy photochemical efficiency $(\mathrm{PEc} ; \mathrm{Fv} / \mathrm{Fm})$ with leaf electrolyte leakage (EL; D) in 18 varieties from tall fescue (TF), chewings fescue (CF), perennial ryegrass (PRG), and kentucky bluegrass (KBG). **Significance of the linear regression at $P=0.01$. tolerance was the greatest in $\mathrm{CF}$, the least in $\mathrm{KBG}$, and intermediate in TF and PRG. 'Ebony' had greater ultraviolet-B tolerance relative to the other four TF varieties. Among seven KBG varieties, 'BlueTastic', 'BlueRiffic', and '747' had better ultravioletB tolerance. Previous studies have shown that plant ultraviolet-B tolerance is closely associated with genetic, physiological, and morphological characteristics (Bjorn, 2004; Ervin et al., 2004b; Mackerness, 2000; Zhang et al., 2005). Plants possess various constitutive and inducible defense mechanisms such as pigment and antioxidant systems for protection against stresses such as ultraviolet-B (Ervin et al., 2004b). It has been documented that KBG varieties with higher levels of pigments and antioxidants had greater ultraviolet$\mathrm{B}$ tolerance than those with less pigments and antioxidants (Ervin et al., 2004b; Zhang et al., 2005). Ultraviolet-B stress may trigger responses of ROS species (such as hydrogen peroxide) and hormones (such as ethylene, salicylic acid), which may signal initiation of defense mechanisms against ultraviolet-B damage (Bjorn, 2004; Surplus et al., 1998). It is also possible that the narrower leaves and thick waxy cuticles found in $\mathrm{CF}$ contribute to its greater ultraviolet-B tolerance relative to $\mathrm{KBG}$.

A significant positive correlation was found between turf quality and PEc, and negative correlations were found between turf quality and EL and SOD activity. Turfgrass species or varieties with less EL and greater PEc generally have greater ultraviolet-B survival relative to the ones with greater EL and less PEc under ultraviolet-B stress. In general, species with greater visual quality may have less SOD activity. The initial level and relative changes of SOD activity in response to ultraviolet-B stress may be closely associated with ultraviolet-B tolerance of different species. UltravioletB-tolerant CF had greater initial SOD activity and exhibited relatively smaller and slower upregulation in response to ultraviolet-B stress when compared with the other three species (Fig. 1D). This suggests that CF may have greater initial antioxidant capacity to cope with ROS production effectively, whereas the other species may need greater increases in SOD activity to suppress ROS toxicity under ultraviolet-B stress. Other defense mechanisms such as pigments, other antioxidants, and hormones may be involved in ultraviolet-B tolerance (Bjorn, 2004; Zhang and Ervin, 2005). The data suggest that PEc, EL, and SOD activity may be used as important physiological parameters in screening varieties for ultraviolet-B tolerance. As incident ultraviolet-B levels continue to rise as a result of stratospheric ozone depletion, turfgrass breeders, growers, and practitioners may want to consider selecting dark green species or varieties with greater $\mathrm{PEc}$, cell membrane integrity (less EL), and stronger ROS scavenging systems.

\section{Literature Cited}

Banowetz, G.M., K.P. Dierksen, M.D. Azevedo, and R. Stout. 2004. Microplate quantification 
of plant leaf superoxide dismutase. Anal. Biochem. 332:314-320.

Bjorn, L.O. 2004. Ultraviolet effects, p. 285-294. In: Nooden, L.D. (ed.). Plant cell death processes. Elsevier Academic Press, San Diego, CA.

Bolhar-Nordenkampf, H.R. and G. Oquist. 1993. Chlorophyll fluorescence as a tool in photosynthesis research, p. 193-206. In: Hall, D.O., J.M.O. Scurlock, H.R. Bolhar-Nordenkampf, R.C. Leegood, and S.P. Long (eds.). Photosynthesis and production in a changing environment: A field and laboratory manual. Chapman \& Hall, London, UK.

Bornman, J.F., S. Reuber, Y.P. Cen, and G. Weissenbock. 1997. Ultraviolet radiation as a stress factor and the role of protective pigments, p. 157-170. In: Lumsden, P. (ed.). Plant and UV-B: Responses to environmental damage. Cambridge University Press, Cambridge, UK.

Caldwell, M.M., R. Robberecht, and S.D. Flint. 1983. Internal filters: Prospects of UV-acclimation in higher plants. Physiol. Plant. 58:445-450.

Costa, H., M. Susana, S.M. Gallego, and M.L. Tomaro. 2002. Effect of UV-B radiation on antioxidant defense system in sunflower cotyledons. Plant Sci. 162:939-945.

Ervin, E.H., X. Zhang, and J.H. Fike. 2004a. Ultraviolet B radiation damage on kentucky bluegrass, II: Hormone supplement effects. HortScience 39:1471-1474.

Ervin, E.H., X. Zhang, and J.H. Fike. 2004b. Ultraviolet $\mathrm{B}$ radiation damage on kentucky bluegrass, III: Cultivar effects. HortScience 39:1475-1477.

Heckman, N.L., G.L. Horst, R.E. Gaussoin, and K.W. Frank. 2001. Storage and handling characteristics of trinexapac-ethyl treated kentucky bluegrass sod. HortScience 36:1127-1130.

King, J.W., J.B. Beard, and P.E. Rieke. 1982. Factors affecting survival of kentucky blue- grass sod under simulated shipping conditions. J. Amer. Soc. Hort. Sci. 107:634-637.

Lingakumar, K., P. Amudha, and G. Kulandaivelu. 1999. Exclusion of solar UV-B $(280-315 \mathrm{~nm})$ radiation on vegetative growth and photosynthetic activities in Vigna unguiculata L. Plant Sci. 148:97-103.

Mackerness, S.A.H. 2000. Plant responses to ultraviolet-B (UV-B: 280-320 nm) stress: What are the key regulators? Plant Growth Regulat. $32: 27-39$.

National Turfgrass Evaluation Program. 2008. Percent winterkill ratings of bermudagrass cultivars, 2003-06. 15 Jan. 2008. <http://www.ntep. org $>$.

Noorudeen, A.M. and G. Kulandaivelu. 1982. On the possible site of inhibition of photosynthetic electron transport by ultraviolet-B (UV-B) radiation. Physiol. Plant. 55:161-166.

Rao, M.V., G. Paliyath, and D.P. Ormrod. 1996. Ultraviolet-B and ozone-induced biochemical changes in antioxidant enzymes of Arabidopsis thaliana. Plant Physiol. 110:125-136.

SAS Institute Inc. 2001. SAS version 8e for windows. SAS Institute Inc., Cary, NC.

Schmidt, R.E. and X. Zhang. 2001. Alleviation of photochemical activity decline of turfgrasses exposed to soil moisture stress or UV radiation. Int. Turfgrass Res. J. 9:340-346.

Smirnoff, N. 1995. Antioxidant systems and plant response to the environment, p. 217-244. In: Smirnoff, N. (ed.). Environment and plant metabolism: Flexibility and acclimation. BIOS Scientific Publ., Oxford, UK.

Smith, P.K. 1985. Measurement of protein using bicinchoninic acid. Anal. Biochem. 150:7685.

Strid, A. and R.J. Porra. 1992. Alterations in pigment concentration in leaves of Pisum sativum after exposure to supplementary UVB. Plant Cell Physiol. 33:1015-1023.
Surplus, S.L., B.R. Jordan, A.M. Murphy, J.P. Carr, B. Thomas, and S.A.H. Mackerness. 1998. Ultraviolet-B-induced responses in Arabidopsis thaliana: Role of salicylic acid and reactive oxygen species in the regulation of transcripts encoding photosynthetic and acidic pathogenesis-related proteins. Plant Cell Environ. 21:685-694.

Systat Software, Inc. 2007. Sigmaplot. Systat Software, Inc., San Jose, CA.

Takeuchi, Y., R. Fukumoto, H. Kasahara, T. Sakaki, and K. Mitsutoshi. 1995. Peroxidation of lipids and growth inhibition induces by UV-B irradiation. Plant Cell Environ. 14:566-570.

Teramura, A.H., J.H. Sullivan, and L.H. Ziska. 1990. Interaction of elevated ultraviolet B radiation and $\mathrm{CO}_{2}$ on productivity and photosynthetic characteristics in wheat, rice, and soybean. Plant Physiol. 94:470-475.

Teramura, A.H. and L.H. Ziska. 1996. Ultraviolet$B$ radiation and photosynthesis, p. 435-450. In: Baker, N.R. (ed.). Photosynthesis and the environment. Kluwer Academic Publishers, Dordrecht, The Netherlands.

Tevini, M. and A.H. Teramura. 1989. UV-B effects on terrestrial plants. Photochem. Photobiol. 50:479-487.

Zhang, X. and E.H. Ervin. 2005. Effects of methyl jasmonate and salicylic acid on UV-B tolerance associated with free radical scavenging capacity in Poa pratensis L. Intl. Turfgrass Soc. Res. J. 10:910-915.

Zhang, X., E. Ervin, and R.E. Schmidt. 2003. Plant growth regulators can enhance the recovery of kentucky bluegrass sod from heat injury. Crop Sci. 43:952-956.

Zhang, X., E.H. Ervin, and R.E. Schmidt. 2005. The role of leaf pigment and antioxidant levels in UV-B resistance of dark- and light-green kentucky bluegrass cultivars. J. Amer. Soc. Hort. Sci. 130:836-841. 